\title{
A rare cause of abdominal pain: Ectopic ovary and intestinal malrotation
}

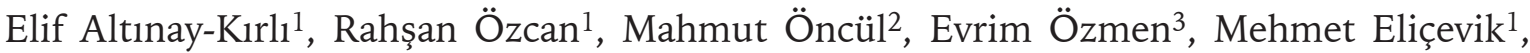 \\ Cenk Büyükünal ${ }^{1}$, Haluk Emir ${ }^{1}$, Gonca Topuzlu-Tekant ${ }^{1}$ \\ ${ }^{1}$ Division of Pediatric Urology, Department of Pediatric Surgery, and Departments of ${ }^{2}$ Obstetrics and Gynecology and \\ ${ }^{3}$ Radiology, Istanbul University Cerrahpasa Faculty of Medicine, Istanbul, Turkey. E-mail: dr.elif@gmail.com \\ Received: 2nd April 2017, Revised: 4th May 2017, Accepted: 19th May 2017
}

\begin{abstract}
SUMMARY: Altınay-Kırlı E, Özcan R, Öncül M, Özmen E, Eliçevik M, Büyükünal C, Emir H, Topuzlu-Tekant G. A rare cause of abdominal pain: Ectopic ovary and intestinal malrotation. Turk J Pediatr 2017; 59: 699-703.

Ectopic ovary is a rare anomaly that can be associated with unicornuate uterus and renal anomalies. Intestinal rotational anomalies are failure of normal rotation and this arrest in development can predispose to develop a malfixated midgut that is a risk factor for volvulus and significant morbidity and mortality especially in early childhood. Cyclic abdominal pain is a common symptom for both of two distinct pathologies in adolescent ages. Here, we report a case of unicornuate uterus together with right ectopic ovary and intestinal malrotation.
\end{abstract}

Key words: ectopic ovaries, midgut malrotation, unicornuate uterus, cyclic pain.

Ectopic ovaries are characterized by the attachment of the upper pole to an area above the level of the common iliac vessels and this condition maybe associated with unicornuate uterus. ${ }^{1}$ Intestinal rotational anomalies are failure of normal rotation and fixation of any portion of the intestinal tract during embryonic development. This arrest in development can predispose to develop a malfixated midgut that is a risk factor for volvulus and significant morbidity and mortality. Cyclic abdominal pain is a common symptom for both of two distinct pathologies in adolescent ages. ${ }^{2,3}$ The purpose of this paper is pointing out presence of togetherness of two different embryological processes in a case and review of similar cases in literature.

\section{Case Report}

A 15-year-old girl was examined with the complaint of cyclic abdominal pain, nausea and non-bilious vomiting. She had regular menstrual cycles since menarche at age 13 . She did not have any history of previous illnesses or constipation. Also she was completely asymptomatic in the painless period. During exacerbations of pain, her physical examination was not compatible with any surgical emergency and laboratory findings were normal. Ultrasound (US) revealed a cystic lesion in the right lobe of the liver, approximately 45 $\mathrm{mm}$ in size, inferolateral to the gallbladder. She also had a pelvic kidney on the right side. Preliminary diagnosis was a duplication cyst. An upper gastrointestinal contrast study showed midgut malrotation instead of the cyst (Fig. 1). Magnetic resonance imaging revealed right ectopic ovary under the right hepatic lobe. It also confirmed the presence of right pelvic ectopic kidney, unicornuate uterus and thoracolumbar scoliosis (Fig. 2).

Family and child were informed about possible diagnoses and treatments, and then informed consent was obtained before the surgery. A combination of intestinal malrotation and undescended ovary lead us to perform laparotomy through a lower midline incision with the aim of surgical correction of both. The right ovary was found buried deep under the posteroinferior segment of the right lobe of the liver. Retroperitoneal vascular connections and a cord like structure, which originated from the right inguinal canal, provided blood supply to the right ovary and fimbria. The ovaries, fimbriae and left Fallopian tube were normal. The right Fallopian tube, on the other 
Table I. Cases of Undescended Ovary in Childhood Period.

\begin{tabular}{|c|c|c|c|c|c|}
\hline Author & Age & Mullerian anomalies & Renal anomalies & Affected side & Means of diagnosis \\
\hline Kives et $\mathrm{al}^{3}{ }^{3}$ & $13 \mathrm{yrs}$. & Bicornuate uterus & None & Bilateral & CT, LS \\
\hline $\begin{array}{l}\text { Trinidad et } \\
\text { al. }{ }^{6}\end{array}$ & 14 yrs. & Vaginal agenesis & $\begin{array}{l}\text { Ipsilateral renal } \\
\text { agenesis }\end{array}$ & Left & MRI \\
\hline Suh et al. ${ }^{7}$ & $14 \mathrm{yrs}$. & Left unicorn uterus & None & Right & MRI, US, LS \\
\hline Present Case & 15 yrs. & Unicornuate uterus & $\begin{array}{l}\text { Right pelvic } \\
\text { kidney }\end{array}$ & Right & MRI, LS \\
\hline
\end{tabular}

CT: computed tomography; LS: laparoscopy; MRI: magnetic resonance imaging; US: ultrasonography

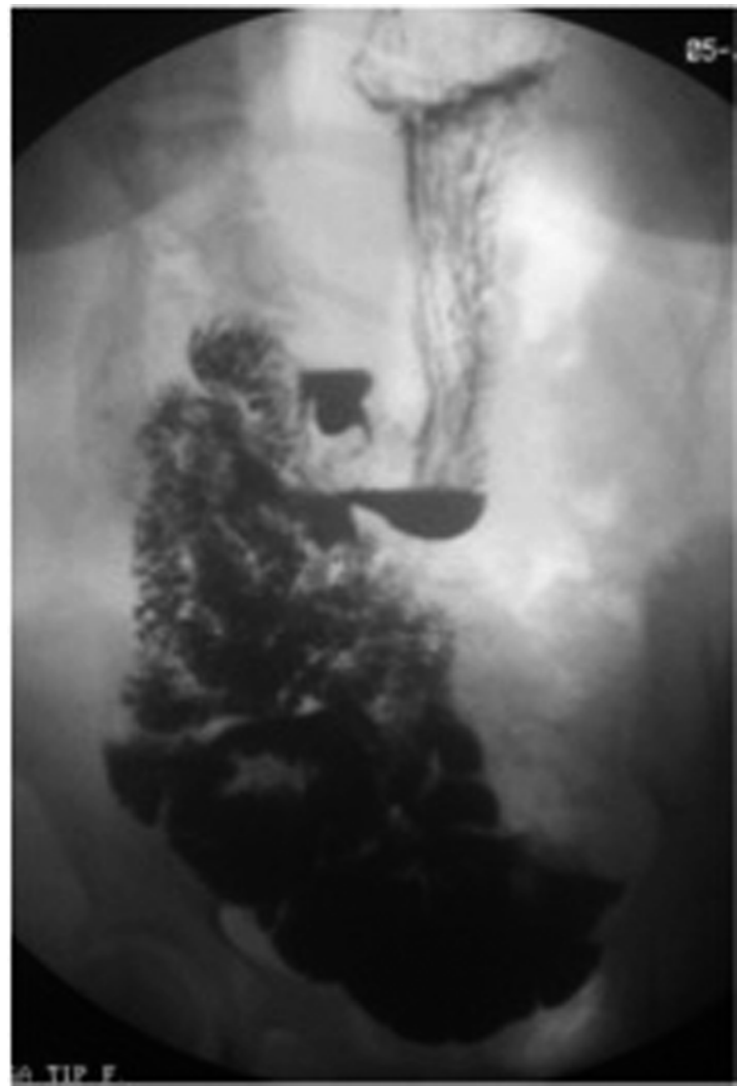

Fig. 1. Upper gastrointestinal contrast study. Absence of duodenum's C curve and descending small intestinal segments from right side of the vertebral column.

hand, was not found to communicate with the right ovary and the patient had a unicornuate uterus and right pelvic kidney.

On surgical exploration, an abnormal configuration of the duodenal C-loop, midlower quadrant located cecum and presence of peritoneal Ladd bands were defined. These findings confirmed the diagnosis of intestinal malrotation in the preoperative radiological evaluation. Excision of Ladd bands, widening of mesenteric root and appendectomy were performed and the cecum was placed in the left lower quadrant as described in Ladd's procedure. ${ }^{3}$ Due to the fact that the vascular structures did not have sufficient length, fixation of the right ovary and fimbria to the posterior abdominal wall were performed to prevent a potential future torsion (Fig. 3).

\section{Discussion}

Undescended ovary is described as attachment of the upper pole of the ovary to any part of the abdominal wall or an organ above the level of the common iliac vessels. It has a rare occurrence with an incidence of $0.3-2 \%$ and it may be unilateral or bilateral. ${ }^{4}$ Gonads are initially located on the medial surface of the urogenital ridge, which is located on the posterior wall of the peritoneal cavity on each side of the spine, during $5^{\text {th }}$ week of development. In the third month of fetal life, ovaries migrate by using chemotactic mechanisms and take their final position between utero-ovarian and infundibulopelvic ligament in the ovarian fossa. Gubernaculum connected to the lower pole of the gonad, guides the ovary during descent to the normal pelvic position. Also gubernaculum attaches the ovary to the uterus and forms the uteroovarian and round ligaments of the uterus. Infundibulopelvic ligament is formed by elongation of suspensory ligament, which is attached to the upper ovarian pole. It has been suggested that problems associated with a short infundibulopelvic ligament, mesovarium and an elongated utero-ovarian ligament or lack of descent or growth restriction of the genital ridge may cause failure of descent of the ovary. The mechanism underlying the undescended ovary is still unclear and when accompanying Mullerian anomalies are taken into consideration multifactorial polygenic 


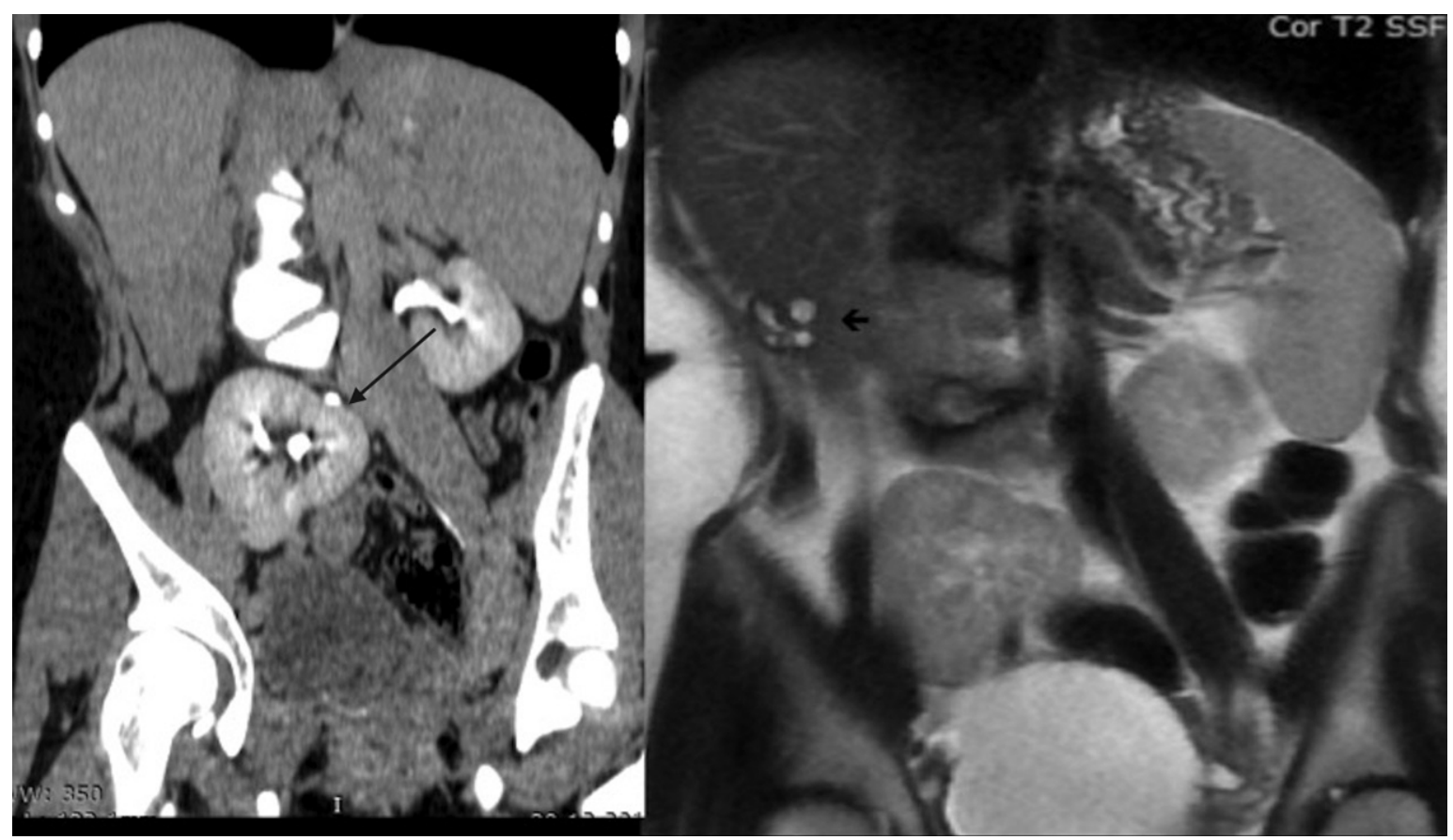

Fig. 2. MRI of ectopic ovary and pelvic kidney: A) Right ectopic ovary under the right hepatic lobe. B) Right pelvic ectopic kidney.

processes can be responsible for developmental insult. ${ }^{1,4,5}$ Combined defects in Wolfian and Mullerian duct may cause Mullerian anomalies such as unicornuate uterus and renal agenesis is commonly associated with undescended ovary. Fallopian tube on the effected side of undescended ovary may be elongated and rudimentary and usually is not attached to the uterus. ${ }^{1}$

Malrotation effects $0.2-1 \%$ of normal population and poses a great risk for midgut volvulus that may lead to short bowel syndrome and death secondary to complications. ${ }^{2,3}$

Embryological explanation of these two distinct processes is completely different and clinical presentation can cause diagnostic dilemmas. ${ }^{2,3,4}$ There are only 3 similar pediatric cases reported in the literature and data related to these cases are summarized in Table I. 5,6,7 Our patient presented with cyclic abdominal pain and was diagnosed with a right undescended ovary, right pelvic kidney and unicornuate uterus, midgut malrotation and scoliosis.

The descent of the ovary appears to depend on the proper development of Mullerian system and undescended ovary and Fallopian tubes anomalies are commonly seen with uterine and renal anomalies. ${ }^{1}$ Mayer-Rokitansky-Kustner-
Hauser syndrome which is characterized with absence of uterus, cervix and renal agenesis association between undescended ovary is reported with an incidence of $20 \% .^{4}$ Unicornuate uterus, which is also a finding in our case, is very common but we did not identify a specific syndrome in our case. ${ }^{4,6}$

Most of cases are asymptomatic and one has been diagnosed during evaluation for primary amenorrhea. Symptomatic patients, who were admitted to hospital with abdominal pain and had surgery, are reported in the literature. ${ }^{7}$ Older aged patients were diagnosed when they were being investigated for infertility ${ }^{1}$.

US is the first line of the radiological examination but magnetic resonance imaging (MRI) is more sensitive and specific to genitourinary system and gives more detail about the anatomy. ${ }^{4}$ In some reports, especially patients with bilateral undescended ovaries had colon and appendix anomalies but midgut malrotation was not identified and none of these cases had vertebral anomalies. ${ }^{5}$ In our case emergency US evaluation defined a thick walled cystic mass without a solid component. Interpretation of this radiological finding combined with non-bilious voiding, may be related with a duodenal obstruction secondary to an intestinal 


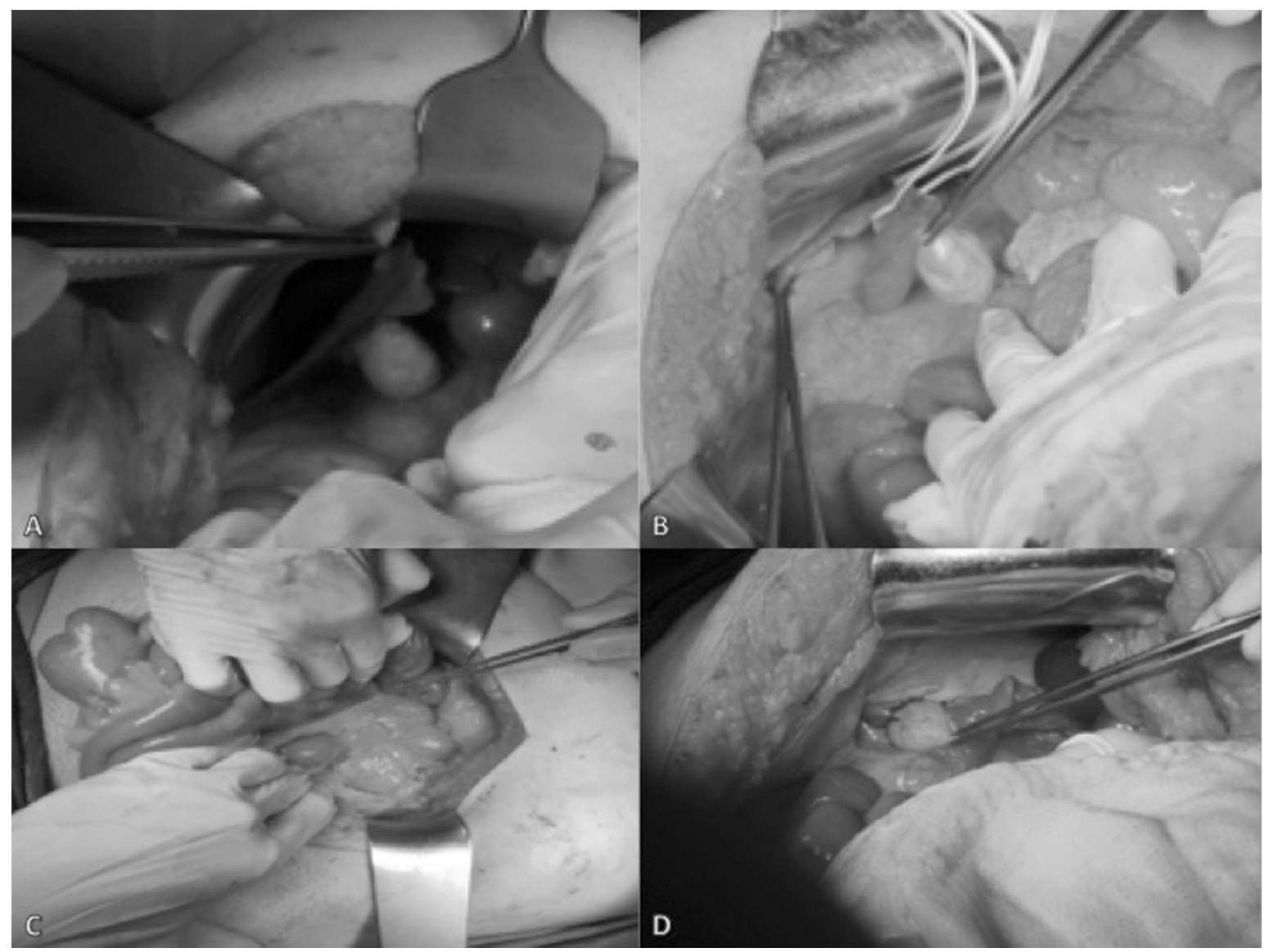

Fig. 3. A) Right ovary has short pedicle and located under liver. B) Release of right ovary and fixation to the lateral abdominal wall at the level of iliac bone with preservation of short pedicle. C) Fixation of intestinal malrotation with Ladd's procedure. D) Final position of the right ovary and fimbria on the posterior abdominal wall

duplication cyst. Upper gastrointestinal contrast study is the first line radiological method for identifying an underlying obstructive pathology in this situation. Midgut malrotation instead of intestinal duplication cyst can also present with cyclic abdominal pain and vomiting in the late childhood period.

There is still a disagreement about the indication of surgery in asymptomatic intestinal malrotation, but most of authors suggest that patients with malrotation after 2 years of age should undergo Ladd's procedure due to risk of delayed diagnosis and complications of volvulus. ${ }^{2}$ In our case cyclic abdominal pain may be associated with intestinal malrotation or secondary to ovarian pathologies. Regardless of the underlying cause of abdominal pain, we chose to investigate the origin and perform correction surgically.

Publications suggest that conservative management should be preferred for an ectopic ovary because there is no association reported between premature ovarian failure or any other gynecological anomaly but it has the risk of ectopic pregnancy. ${ }^{4}$ Acute abdomen secondary to a corpus luteum cyst in an ectopic ovary was previously reported and it can be confusing for the surgeon because of the anatomic anomaly. ${ }^{5}$ Fertility problems may be the concern of these patients in the future. Spontaneous pregnancy is reported in the literature with an oocyte produced from an ectopic ovary by transperitoneal gamete migration. ${ }^{1}$ Presence of a uterine anomaly with one healthy, normally located ovary and tube can receive fertility treatment with intra uterine insemination. ${ }^{8}$ Besides different in vitro fertilization techniques have been used in the past and resulted with successful pregnancies. ${ }^{4}$ If associated Mullerian anomalies are present, 
miscarriage, ectopic pregnancy, and abnormal fetal presentation are much more common in this population. Patients should be warned for related problems. ${ }^{8}$

Undescended ovaries are uncommon and associated especially with genitourinary anomalies. One of the underlying causes of abdominal pain is intestinal malrotation and can be mortal. Both of these pathologies cause cyclic abdominal pain and are impossible to distinguish which is the cause. Symptomatic intestinal malrotation must be corrected surgically but it is not necessary to remove ectopic ovary or tube unless there is the suspicion that tube can cause ectopic pregnancy in the future. Patients should be counselled about the risk of future fertility.

\section{REFERENCES}

1. Ombelet W, Deblaere K, Grieten M, et al. Intrauterine pregnancy following transperitoneal oocyte and/ or sperm migration in a woman with an ectopic (undescended) ovary. Reprod Biomed Online 2003; 7: 110-113.
2. Graziano K, Islam S, Dasgupta R, et al. Asymptomatic malrotation: Diagnosis and surgical management; An American Pediatric Surgical Association outcomes and evidence based practice committee systematic review. J Pediatr Surg 2015; 50:1783-1790.

3. Adams SD, Stanton MP. Malrotation and intestinal atresia. Early Hum Dev 2015; 90:921-925.

4. Dietrich JE, Hertweck SP, Bond S. Undescended ovaries: A clinical review. J Pediatr Adolesc Gynecol 2007; 20:57-60.

5. Kives SL, Perlman S, Bond S. Ruptured hemorrhagic cyst in an undescended ovary. J Pediatr Surg 2004; 39: e4-e6.

6. Trinidad C, Tardaguila F, Fernandez G, et al. Ovarian maldescent. Eur Radiol 2004; 14: 805-808.

7. Suh DS, Han SE, Yun KY, et al. Ruptured hemorrhagic corpus luteum cyst in an undescended ovary: A rare cause of acute abdomen. J Pediatr Adolesc Gynecol 2015; 30: 1-4.

8. Ombelet W, Verswijvel G, Vanholsbeke C, et al. Unicornuate uterus and ectopic (undescended) ovary. Facts Views Vis Obgyn 2011; 3: 131-134. 